\title{
Nada está en nada. 0 todo el mundo sabe la lógica. \\ El método de enseñanza universal de Joseph Jacotot y la emancipación intelectual en las clases pobres ${ }^{1}$
}

\author{
Dr. Patrice Vermeren²
}

"Antes de entrar en materia, declaro que no veo en usted más que un curioso; por lo tanto, lo pongo en cuarta línea. Si viniera un pobre, un campesino, un padre de familia, él pasaría antes que usted". En estos términos es recibido en Lovaina, un día de 1829, el enviado de la prestigiosa Sociedad de Métodos de París, llegado a informarse ante el inventor de los resultados de un método de Enseñanza universal del que el mundo sabio y los proletarios hicieron gran ruido 3 . El abogado Baudoin, al que el ministro francés de Instrucción Pública

1 Traducción de la Dra. Carolina Ávalos Valdivia, chilena, Doctora en Filosofía por la Université Paris 8 Vincennes-Saint-Denis y por la Pontificia Universidad Católica de Valparaíso. Académica de la Université de Technologie de Compiègne, Campus Viña del Mar, Chile. Contanto: carolina_avalos_valdivia@yahoo.com

2 Académico del Departamento de Filosofía de la Université Paris 8 Vincennes - SaintDenis. Este texto forma parte de la publicación colectiva Les sauvages de la cité. Auto-émancipation du peuple et instruction des prolétaires au XIXe siècle. Seyssel: Champ Vallon, 1985. Con prólogo de Jacques Derrida, la presentación de Jean Borreil y textos de Stéphane Douailler, Jean-Claude Pompougnac, Jacques Rancière, entre otros. Esta publicación es el resultado de las discusiones que se dieron en el Coloquio Les savoirs populaires, I'instruction des prolétaires et la philosophie des clases pauvres au XIXe siècle, llevado a cabo en Creusot, Francia los días 6 y 7 de octubre de 1984. La dirección estuvo a cargo de Jacques Derrida y la coorganización de Patrice Vermeren (Collège International de Philosophie), Daniel Puymèges (Institut Jean-Baptiste Dumay), Jean Borreil y Jacques Rancière (Groupe d'Etudes sur les Répresentations du Social, C.N.R.S.) y por Rose-Marie Ferenczi (Ecole de Hautes Etudes en Sciences Humaines et Sociales). Contacto: vermeren.patrice@gmail.com

3 Boutmy, E. Considérations sur les résultats importants qu'obtient en Belgique le nouveau mode d'éducation inventé par $M$. Jacotot adressées aux Péres de famille et à toutes les presonnes qui s'occupent d'éducation. Paris, 1829. 5. 
ha encargado redactar un informe sobre el mismo tema, describe en términos idénticos la recepción que le ofreció Joseph Jacotot, y el placer maligno que esto provoca al desconcertar a aquellos que tal vez piensan avergonzarlo:

Estaba en posición de ver, en efecto, que en la amabilidad con la cual el señor Jacotot recibe a las personas que llegan hasta él, se mezcla en ocasiones alguna cosa inusitada, resultado de su posición. No habiendo encontrado muy seguido, en los visitantes recurrentes, más que indiscretos conducidos por una curiosidad secreta y estéril, o incluso algunos no para acudir a él, sino para darle lecciones, no para aclarar sus dudas, sino para dictar sus decisiones, no sabe, por cada nuevo llegado, si él no debe atenerse a semejantes impertinencias. En esos primeros momentos, parece no estar ocupado más que en adivinarlos; los mira hasta el fondo del alma: ustedes dirían que disfruta ante todo de lo que sus principios tienen de más audaz, y, más, en posición con las ideas recibidas; como si él quisiera proporcionar a sus secretas disposiciones, una oportuna ocasión de estallar, y, golpeándolos de frente, llegar repentinamente a conocer si la verdad no estuvo de parte de vuestro criterio más que del deseo de instruirlos ${ }^{4}$.

\section{Hacer y dejar decir}

Esta entrada en materia es ritualmente seguida de una visita en los internados de la señorita Marcélis y del señor Deschuyffeleer, que aplican el método en toda su pureza. El visitante es invitado a dar sus palabras a los alumnos, sobre las cuales ellos improvisan una composición: el conde de Lasteyrie propuso "incidente" y

4 Baudoin, F. M. Rapport sur les résultats, l'esprit et l'influence morales et intellectuelle de la méthode de M. Jacotot présenté à $M$. De Vatimesnil, ministre de l'instruction publique, le 5 août 1829, et dédié aux pérres de famille. Paris, 1829. 3-4. 
"vagabundaje", otros eligen "convención" y "la verdadera cortesía". Sobre este último tema, a catorce años y después de dos años de estudios, en un cuarto de hora y a pesar del ruido, una joven persona entrega este escrito: "Seguir las convenciones del mundo, ser amable para tener reputación, ser complaciente con aquellos de los cuales esperamos complacencia, aquí está lo que continuamente en la sociedad se entiende por cortesía; pero no está allí, sin embargo, en qué consiste lo verdadero. La verdadera cortesía es aquella que se ejerce indiferentemente entre el rico y el pobre, aquella que es natural, aquella finalmente que no está dictada más que por el sentimiento del bien. El orgullo y la ambición no entran en absoluto en el corazón de aquel que posee la verdadera cortesía. Siempre bueno con sus inferiores, los alienta, siempre amable con sus iguales, se asegura su amistad; siempre franco pero respetuoso con sus superiores, él gana su confianza, finalmente, se hace amigos en todas partes. Una instrucción sólida es el germen de la verdadera cortesía; ella proviene también de la educación. La verdadera cortesía consiste en conocer las conveniencias sociales, seguir las convenciones del mundo, no obstante sin adoptar los prejuicios; ella es la complacencia sin bajeza, la amabilidad acompañada de gracias; en una palabra, ella debe partir del alma, ser el lenguaje del corazón". El pedagogo invita luego a comparar con lo que podría escribir, al mismo tiempo, un literato de París. El mismo ejercicio con la música: se trata de improvisar, sobre un texto de un poema tomado al azar, la redacción de una partitura, que se ejecuta en solitario, en dúo, en coro, en el acto, con el acompañamiento del piano. El incrédulo se va convencido, el adepto triunfa. El pedagogo vuelve a su trabajo y a su destino: hay que hacer y dejar decir.

Lo que fascina a los invitados de Jacotot es, en principio, este dominio de la lengua escrita en los niños analfabetos algunos meses antes, esta capacidad de componer y ejecutar una melodía de parte

5 Carta de Jacotot del 24 de Agosto de 1828 (Archives du Musée National de I'Éducation, 39, rue Lacroix-Vaubois, 76130 Mont-Saint-Aignan) comunicada por Serge Chassagne. 
de neófitos en los que el origen social es evidentemente proletario. Ahora bien, la pregunta original de la elite progresista de la Restauración, en 1829, es precisamente aquella de la educación del pueblo. El ministro de la Instrucción Pública, el señor de Vatimesnil, que restituye a la Universidad el poder de nominación y revocación de los institutores, prohibió el método de la enseñanza individual: "es defectuoso en todos los puntos, consume de forma miserable el tiempo de los niños, es causa de tantos abusos y desórdenes, que no sabríamos llegar a suprimirlo rápidamente en el doble interés de la instrucción y de las costumbres" ${ }^{6}$. Cuatro quintos de los institutores primarios, lamentablemente, la practican. El método simultáneo, en uso en establecimientos de hermanas cristianas, es percibido como rutinario y favorable a los objetivos oscurantistas de los Ultras y del clero. El método mutuo, finalmente, que conoció su hora de gloria entre 1815 y 1820 y [que] gozaba aun ante los liberales del aura de la persecución en la que había sido víctima desde entonces, es crédito de la economía del personal, que permite realizar por sustitución de los alumnos más sabios y los mejor dotados (los monitores) por los sub-maestros, aunque criticado por su modo mecánico de transmisión de conocimientos, su disciplinarización ultrajada de la relación pedagógica y su poca eficacia. El rumor de un método nuevo, mientras que "el desánimo era tal que no se formaba más a ningún institutor, ninguna institutriz en el estudio de los métodos perfeccionados"7, y por su pretensión de dirigirse a los pobres y a los ignorantes más que a la nación sabia ${ }^{8}$, intriga y seduce a filántropos ilustrados y proletarios autodidactas que quieren emancipar al pueblo o reformar la sociedad.

\footnotetext{
6 Circulaire du ministre de l'instruction publique relative aux méthodes que doivent suivre les instituteurs primaires, et aux brevets dont ils doivent être pourvus del 31 de enero de 1829, Archivos nacionales, F17.

7 Ver de Gérando, sesión del 20 de abril de 1828 de la sociedad de la instruccion elemental, citado por Maurice Gontard, La Question des écoles normales primaires de la Révolution de 1789 à 1962. Toulouse: CRDP, 1975. 21.

8 Jacotot, Joseph. Prólogo a la cuarta edición de L'enseignement universal, langue maternelle. Paris, 1892. XIX.
} 


\section{El descubrimiento del método}

Si el nombre de enseñanza universal puede ser fechado de forma precisa el 15 de octubre de 1818, el descubrimiento del método que designa procede de una experiencia profesional muy anterior y complicada. Jacotot, el mayor de nueve hermanos, hijo de un comerciante carnicero de Dijon, había seguido estudios brillantes gracias a Joseph Tardy, su abuelo materno, por su condición de maestro carpintero. La revolución hace de este abogado de veintiún años, profesor de Humanidades en el Colegio de Dijon durante dos años, un capitán de artillería, luego un director de la oficina de pólvora y salitre, en reemplazo de Fourcroy, finalmente, un sustituto del director de la escuela politécnica, puesto prestigioso que abandona para ocupar la cátedra de Lógica y de análisis de percepciones e ideas en la primera Escuela central de su ciudad natal. Su curso de Método de las ciencias (se sabe que los Ideólogos habían expulsado la palabra "filosofía" de los programas) parece autorizarse en vista del comité de la Instrucción Pública y del informe de Daunou a la Convención: Libertad de métodos instructivos ${ }^{9}$, para alejarse de la ratio studiorum de los jesuitas y del compendium philosophiae inspirado de la filosofía de Lyon; el profesor indica el tema al inicio de la sesión, propone un orden metódico de la discusión y deja a los alumnos la palabra que no retomará sino al final de la lección, para poner en forma una síntesis de las ideas y de los argumentos ${ }^{10}$. Desde 1795, Jacotot manifiesta —en la misma forma de su enseñanza - que la verdad está del lado del alumno más que del maestro; él ya considera al Telémaco de Fenelón como la mejor lectura educativa, como muestra su alumno Etienne Cabet ${ }^{11}$, quien verá allí una opinión favorable para la Comunidad y su primera utopía. Como secretario del comité de profesores, redacta un proyecto de curso, apuntando a reducir la brecha constatada entre los estudios primarios y los de la central, y para reanimar la instrucción pública a través de la emulación "que dio a luz en los griegos y en los romanos

9 Ídem.

10 Ver Guillard, Achille. Biographie de Joseph Jacotot. Paris, 1860. 14.

11 Carta de Jacotot al diputado Dupont, 1830, Archivos nationales, F17 20984. 
tantos genios felices y de talentos sublimes"12. Se sabe también, por la respuesta que envía a la consulta del Ministro del Interior sobre los métodos recomendados por los manuales y utilizados por los maestros en $1799^{13}$, que enseña las lenguas antiguas por analogía: "yo no digo en absoluto a mi alumno que 'copia' quiere decir 'abundancia', le digo: usted conoce copioso, por consecuencia copiosus, y de copiosus a copia, no hay más que un paso, etc.". Hay que ir, en todas las materias, de lo conocido a lo desconocido.

Hasta la Restauración, Jacotot acumula títulos (doctorados en Letras, en Derecho y en Ciencias) y funciones (Cátedras de matemáticas trascendentales en el liceo de Dijon en 1804, luego en la Facultad de ciencias en 1809, suplencia del curso de Derecho Romano en la Escuela de Derecho en 1807). Los Cien días lo hicieron Diputado, el regreso de los Borbones provoca su exilio voluntario a Bélgica. Su reputación de sabio, la gran variedad de conocimientos, la eficacia de su enseñanza y su adhesión a los principios filosóficos de la Revolución Francesa, permite que lo nombren Lector de literatura francesa en la Universidad de Lovaina. Pero, ¿cómo se hace escuchar en un auditorio donde la lengua es la holandesa, cuando no la habla él mismo? Pregunta propiamente pedagógica, puesto que requiere la puesta en marcha de un arte de transmisión de saberes - que no sabría hacer economía del dominio del instrumento de su comunicación, pero que compromete también la naturaleza de la materia enseñada - en un discípulo de los Ideólogos, para quien el lenguaje no es solamente el vehículo del pensamiento, sino que participa de su elaboración. La invención del método es la solución encontrada — por azar — para una dificultad conocida en la experiencia ${ }^{14}$ : Jacotot

\footnotetext{
12 Projets de règlements aux écoles primaires et à l'école centrale, Archivos departamentales de la Côte d'Or, L 1082.

13 Carta de Jacotot al ministro de Asuntos interiores, del 16 messidor año 7, Archivos nacionales F 171342.

14 Es una constante de los relatos del descubrimiento de una pedagogía nueva, proyectar en el origen una situación dada por insalvable y superada por la utilización salvaje de una técnica finalizada: ver la invención de la imprenta en la escuela por Célestine Freinet, porque gaseado durante la guerra se agotaba al hablar en clases [Nota del autor].
} 
da a sus alumnos una traducción holandesa del Telémaco de Fenelón y les pide aprender de memoria el texto de la edición francesa. Por la confrontación que ellos hacen de palabras y frases, por la repetición de los primeros capítulos y el relato de los siguientes, adquieren muy pronto las reglas de ortografía y de gramática de la lengua francesa, por ellos mismos y sin la ayuda de ningún maestro. La excelencia de los resultados incita al fundador a aplicar su principio al estudio de la lengua materna, a generalizarlo para el aprendizaje de todas las lenguas extranjeras - muertas o vivas-, a importarlo en la adquisición de todos los otros órdenes de conocimiento: escritura, diseño, pintura, matemática, Derecho, etc. En 1823, publica la Enseñanza universal, lengua materna, que lo hace seguir de la Enseñanza universal, lengua extranjera (1824), de la Enseñanza universal música, diseño y pintura (1824) y de la Enseñanza universal, matemáticas. Sus hijos fundan en 1828 el Diario de la emancipación intelectual, y la polémica se difunde por toda Europa por las virtudes de la doctrina del Sócrates moderno, o contra las charlatanerías de la jacotomachie ${ }^{15} 16$.

\section{Todas las inteligencias son iguales}

El fundamento primero de la enseñanza universal es la igualdad de las inteligencias: las diferencias constatadas de hecho entre el entendimiento de un campesino y el de un aristócrata, entre el de un ignorante y el de un sabio, o el del hombre y de la mujer, no son atribuibles a la naturaleza que ha distribuido en cada individuo capacidades intelectuales idénticas, sino a la educación, desigualmente extendida en la familia y en la sociedad, que proviene de la voluntad

15 Se conserva la palabra en francés, considerando que no existe una traducción literal. La expresión hace referencia a "los trucos de Jacotot" [Nota de la traductora]. 16 Ver Duriveau, M. Examen raisonné de l'enseignement dit universal, qui a pris naissance dans le Pys-Bas. Bruxelles, 1827; Laroche, B. L'enseignement universel de M. Jacotot en présence de l'enseignement universitaire. Paris, 1829; Lorain, L. Réfutation de la méthode Jacotot, considérée dans ses principes, ses procédés et ses résultas. Paris, 1830, et Chompré, La Jacotomachie, ou le Pour ou le Contre de la Méthode Jacotot. Paris, 1829. 
y de la consideración. Para legitimar esta tesis, Jacotot, participante de la tradición filosófica de las clases pobres ${ }^{17}$, recurre a paternidades prestigiosas donde la autoridad es universalmente reconocida: Descartes, Helvétius, Locke y Newton, todos los cuales, según él, profesan esta opinión $n^{18}$. Pero, sobre todo, demuestra que hay muchas razones para no defender la opinión contraria, como aquella que le objeta, por ejemplo, el duque de Lévis.

En una carta hecha pública en 1829, el par de Francia arguyó cuatro órdenes de consideraciones propias, según él, para cuestionar —en nombre de la razón e incluso del simple buen sentido- los principios de un método donde no se cuestiona, por lo demás, ni ingeniosidad ni utilidad: 1 - la observación prueba que las facultades intelectuales son a imagen de las facultades físicas, profundamente desiguales entre los individuos; 2- no hay igualdad sino ante Dios, solo lo infinito hace desaparecer las diferencias; 3- la naturaleza, en su inconcebible variedad, no produce nada semejante, y por consecuencia nada igual; 4- a nivel de las consecuencias, esta doctrina de la igualdad de las inteligencias ya no solo es falsa, es nefasta, porque aumenta la vanidad de la juventud de los dos sexos, trastorna la paz del hogar (¿cómo los matrimonios podrán mantenerse una vez que el amor que se desvanece con la edad haya desaparecido?) y amenaza el orden social, destruyendo en los espíritus testarudos la idea de una superioridad intelectual de las elites dirigentes ${ }^{19}$. Jacotot refuta término a término a su correspondiente: 1 - la tesis de la desigualdad natural de las inteligencias es al menos igual de indescifrable que la suya, y puede ser sostenida al igual que una opinión; 2- ningún medio permite medir a priori una inteligencia, y la diferencia constatada de los resultados puede deberse tanto a la mala voluntad, a la distracción, a las circunstancias en general como a la capacidad intrínseca de los individuos; 3- tenemos el deber, en

17 Ver Vermeren, Patrice "La philosophie populaire", Seminario en el Colegio Internacional de Filosofía, 1983-84.

18 Jacotot, Joseph. L'enseignement universal, langue maternelle, op. cit. Prólogo a la primera edición, p. viij.

19 Duc de Lèvis, carta a M. Jacotot sobre la doctrina de la igualdad de las facultades intelectuales, citado en los anexos de E. Boutmy, op. cit. 72 sq. 
consecuencia, de prodigar a todos la misma educación, como si cada uno tuviera una inteligencia igual; 4- en el ámbito de las consecuencias, lejos de arruinar el orden social y de fomentar al pobre la revuelta, el postulado de la igualdad de la inteligencia implica que ella no depende de los objetos a los cuales ella se aplica: su ejercicio vale tanto en dignidad como cualquier saber o saber hacer que requiera su puesta en práctica, toda jerarquía de valores entre las artes y las ciencias es abolida, cada uno se encuentra satisfecho y feliz de contribuir, desde su lugar, a la felicidad de todos y al perfeccionamiento general de los conocimientos y las técnicas ${ }^{20}$. Jacotot concluye que el genio no existe: no es más, dice, que un duende. Quien quiere, puede.

\section{La autodidaxia contra los maestros explicadores}

Porque el hombre es una voluntad servida por una inteligencia, no nos podemos valer de ningún título para enseñar a otro. La enseñanza universal, en el sentido propio del término, no es una enseñanza, ya que no habría transmisión de un saber, sino solamente estimulación y apoyo a una voluntad deseosa de aprender. Un sabio holandés que fue a investigar a Lovaina en 1826 critica así la denominación del Método: "una denominación que expresaría lo que los griegos nombraban autodidaxis (enseñanza que se da a sí mismo) haría conocer mejor el carácter distintivo del método; porque el maestro de la Enseñanza universal no es más que un guía, que muestra al alumno el camino que debe seguir; le corresponde al alumno caminar solo, y él mismo hacerse hábil en la rama que él cultiva, mirando, reflexionando, y sobre todo repitiendo sin parar lo que ha descubierto y encontrado a través de sus propios esfuerzos en la ciencia a la cual está consagrado. Se parece de alguna manera a un hombre que, sin maestro, sin gramática, sin diccionario, podría aprender una lengua que le es del todo extranjera, y que no tuviera por guía más que una obra original escrita en la lengua que él estudia, acompañado de una traducción; con la

20 Joseph Jacotot, respuesta a M. le duc de Lèvis; ver también el Prólogo a la cuarta dedición de L’Enseignement universal, langue maternelle, op. cit. 
diferencia que cada vez que, en la Enseñanza universal, el maestro, que no debe ser considerado más que un compañero de viaje que hace el camino con el alumno, llama su atención en cada palabra, en cada frase, en cada sílaba, en cada carta en que una frase es compuesta; le hace encontrar las aproximaciones, la fuerza de repetir sin parar y de confiar siempre en su memoria" ${ }^{21}$. Jacotot elabora una pedagogía sin maestros explicadores: estos no deben hacer nada, puesto que es de la naturaleza de la inteligencia humana instruirse sola. Asimismo, no es indispensable saber la materia que se enseña, en Lovaina el fundador pudo enseñar el holandés a sus alumnos aun cuando él no lo hablaba, o enseñar música que él ignoraba a un primer violín de orquesta de Londres. Sócrates disimulaba su saber, el Sócrates moderno puede no saber efectivamente nada. De aquí además la hostilidad que manifiestan en su contra el periodismo y la universidad, donde el estatus y los ingresos están ligados al reconocimiento de su función de explicadores; y cuando los preceptores de los hijos del príncipe de Orange vienen a consultar al filósofo de Lovaina, él responde a su señor que ellos no tienen ninguna disposición para la Enseñanza universal, como Aristóteles había advertido a Felipe, consultado por la educación de Alejandro, de desconfiar de los platónicos ${ }^{22}$.

Todos los intentos por hacer reconocer su método como doctrina oficial —en Bélgica, en Francia- fracasaron. Jacotot deduce de ello que los prejuicios tienen siete vidas y que incluso los reyes no tienen la fuerza para desprenderse de ellos. El fracaso de la Escuela Normal Militar de Lovaina, como la resistencia del Ministerio de la Instrucción Pública en París, lo incitan a buscar en el nuevo mundo la comprensión que no encontró en Europa. Escribe a La Fayette:

Todo hombre que es enseñando no es más que la mitad de hombre. En todas partes donde hay escolares, hay maestros. Cuando la inteligencia no es libre, no veo qué podría serlo. Aviso a los Americanos. Se puede ser independiente sin ser

21 Kinder, J. Rapport sur la méthode de M. Jacotot, presentado al Ministro del Interior del Reino de los Países Bajos el 8 de septiembre de 1826.

22 Carta de M. Jacotot del 24 de agosto de 1828, op. cit. 
libre; la independencia es relativa, la libertad es absoluta. Soy independiente cuando no tengo maestro, es el hecho de otro; soy libre cuando no quiero un maestro, es mi hecho. Para que esta voluntad sea firme, estable, invariable, hay que sentir sus fuerzas, todas sus fuerzas, no solamente morales, sino intelectuales. (...) Hay que trabajar para el futuro, hay que perfeccionar la familia que es la base del orden social. Es necesario que en los hogares se ejerza la inteligencia librándola de toda explicación embrutecedora. (...) Si esta mejora en las familias no realza la nación, es que la perfectibilidad del género humano es una quimera. Si algún día los hombres deben perfeccionarse, no se lo agradecerán a los métodos buenos. Cada uno de nosotros sabe qué es lo que hay que hacer para eso; y desde que un hombre exige un método, un explicador, un maestro, es esclavo, se embrutece. Sería singular que todo esto no sea mejor comprendido en América que en Europa, en fin voy a hacer el intento. Aprovecho entonces vuestra atenta propuesta, y le ruego anticiparse a todo Señor Presidente de Estados Unidos asegurándole mis más profundos respetos ${ }^{23}$.

Washington le envía un hombre joven para instruir, con su propia imagen, pero la experiencia no produce los resultados esperados, a causa de la incomprensión del alumno de la lengua francesa y de su falta de voluntad. La Enseñanza universal se encuentra muy mal y la Emancipación intelectual muy bien: "Cada día nuevas familias participan a beneficio, ricos o pobres, poco importa. Un diputado, un prefecto, un carpintero, yo acojo todo lo que se presenta. Uno de los amiguitos del duque de Bordeaux aprende latín con su institutriz, quien no sabe ni una palabra; un simple obrero estudia diseño, sin maestro explicador, etc." ${ }^{24}$. De vuelta en París después de la Revolución de Julio, Jacotot sigue con la propaganda de su método, particularmente

23 Carta de Jacotot al general La Fayette del 26 de septiembre de 1828.

24 Carta de Jacotot a Serph-Dumagnon del 21 abril de 1831, Archivos del Museo Nacional de la Educación, Mont-Saint-Aignant. 
dirigida a los niños designados por la vieja escuela como incapaces y abandonados por todos los profesores ${ }^{25}$, y a los padres de familia, procurando que sus discípulos no hagan negocio de su descubrimiento y que lo propaguen sin retribución.

\section{Todo está en todo. Se retiene solo lo que se repite}

Cualquiera está dotado de razón, por lo tanto es capaz de captar relaciones. Todo el mundo sabe alguna cosa, y puede compararla con lo que no sabe todavía. Sepa entonces qué es lo que sabe, escribe Jacotot, y lleve consigo todo el resto; conozca un libro, y lleve todos los libros. Si se elije el Telémaco de Fenelón, es en principio por azar; pero también por la oportunidad, porque está escrito en una lengua rica —lexicológica y sintácticamente-, clara y correcta, y porque presenta situaciones variadas y propone un modelo de moralidad. Pero el primer libro podría servir del mismo modo, y llegar a ser la fuente de todos los conocimientos posibles: todo está en todo, entonces nada está en nada.

Nadie duda, escribe Jacotot, que sería muy sabio quien conociera un libro y que supiera todos los comentarios a los que se puede dar lugar. Es verdad que este supuesto es absurdo en el método antiguo; este resultado no puede ser obtenido sino a fuerza de desvelos y años; es el fruto de esfuerzos continuos de una memoria que sucumbe sin cesar bajo la carga de un número prodigioso de hechos y de reflexiones nuevas, dispersas, sin orden, y por consecuencia, sin relación; pero lo que parece imposible se convierte en un juego cuando se comienza por conocer un libro. Es sencillo darse cuenta que todos los otros libros no son otra cosa que el comentario y el desarrollo de las ideas contenidas en el primero. No aprendéis nada sin llevarlo, por el pensamiento, al primer objeto de vuestros estudios: este ejercicio debe durar toda la vida. Éste

25 Ver, L'Enseignement universel mis à la portée de tous les pères de famille par un disciple de J. Jacotot, seconde édition. Paris, 1830, tome 3, 70, note 1. 
se forma así de relaciones íntimas entre vuestras ideas: ellas se ayudan mutuamente, se desarrollan, se aclaran la una a la otra; aunque se toquen por todas partes, no se mezclan. Es un círculo inmenso donde incontables puntos se presentan en el pensamiento uno a uno, si quiere, reunidos o desunidos, en número que él ha fijado, en fin, donde todo el conjunto y los detalles forman un todo que la inteligencia puede abarcar de un solo vistazo ${ }^{26}$.

El sistema del Método descansa entonces en un arte combinatorio de hechos, de ideas y de palabras, puestos en marcha por el alumno a partir de un número finito de pensamientos comunes al género humano que la primera lectura puede entregar a todo el mundo, si es ayudado en su propio proceso por un cuestionamiento en que el modelo explícito es el diálogo socrático. Todo reside por lo tanto en el esfuerzo y la memoria: no se retiene más que lo que se repite. Lo importante no es aprender, sino retener. El único trabajo del maestro, y desde entonces no podemos reconocerle más ese título, es verificar incansablemente que su discípulo ha retenido bien, y comprendido lo que él ha retenido ${ }^{27}$.

Sea el caso de un analfabeto. Su primera lección se desarrolla así:

Ponemos bajo los ojos del alumno el primer libro de Telémaco. Decimos:
Calipso
Calipso no podía
Calipso no podía consolarse por
Calipso no podía consolarse por la partida
Calipso no podía consolarse por la partida de Ulises.

\footnotetext{
26 Citado por B. Pérez, en el artículo Jacotot du Diccionaire de pédagogie et d'instruction primaire de Ferdinand Buisson, Paris, 1888, tomo segundo de la primera parte, p. 1401.

27 Ver la carta de Jacotot a Madame Hennequin del 24 de junio de 1826, Museo Nacional de la Educación, Mont-Saint-Aignan.
} 
El alumno repite. Hacemos escribir esta frase hasta el final según un ejemplo. Se verifica que el alumno distingue todas las palabras, todas las sílabas, todas las letras. Tengan cuidado de ir muy rápido en el comienzo, retengan al alumno en la primera lección hasta que él la sepa imperturbablemente ${ }^{28}$.

A partir de la segunda lección, se comienza a repetir y a verificar. El proceso, según Jacotot, es conforme a la naturaleza: se procede del conjunto al detalle, se termina por el deletreo, en relación a todos los métodos de lectura. La ortografía y la gramática, por constatación de la oreja y de los ojos, llegan rápidamente y por añadidura, sin aprendizaje de reglas a priori. Desde el comienzo, hay que asegurarse que el alumno comprendió bien, para concentrarse en plantearle preguntas que pueda responder por la sola referencia al texto que viene de recitar. Así para la primera frase del Telémaco:

EL PROFESOR pregunta: ¿Quién partió?

EL ALUMNO responde: Ulises.

P. -¿Quién no se puede consolar?

R. -Calipso.

P. - ¿Por qué no se puede consolar Calipso?

R. - Por la partida de Ulises.

P. - ¿Calipso amaba realmente a Ulises?

R. - Sí.

P. -¿Por qué dice eso?

R. -Porque si Calipso no hubiera amado a Ulises, ella no hubiese estado inconsolable después de su partida.

P. -¿Por qué dice inconsolable?

R. -Porque es la misma cosa que no poder consolarse.

P. - ¿Cómo sabe eso?

R. -No sé nada, pero lo creo. 
Es ahora que el maestro debe convencer al alumno a no usar ni una sola palabra, ni una sola expresión, que no pueda mostrar lo que él ha aprendido ${ }^{29}$.

Etc.

Se procederá de la misma manera con el latín, a partir de la Epitome historiae sacrae, con la música (hay que estudiar una partitura y traer todas las otras) o con las matemáticas (toda la aritmética está en una regla de cálculo).

\section{La infancia sometida a ella misma, ¿o el método natural?}

Después de 1834, retirado en Valenciennes y enfermo de una tortícolis que lo obligaba a llevar permanentemente una venda alrededor de la cabeza, Jacotot mantiene una abundante correspondencia con sus discípulos, maestros de institución o padres de familias pobres, aunque ya no intenta dirigirse más a los poderes públicos y parece resignado a una gloria póstuma que vendría de su reconocimiento por la sociedad civi $^{30}$. A pesar de un renombre que sobrepasa Bélgica y Francia —su método fue adoptado por las instituciones de educación de Séprés en Anvers, de Deshouillères y de Frérejean en París, de Guillard en Lyon, y también aquellas de Tourrier en Londres, por el Gimnasio de DeuxPonts en Baviera, la Escuela de cadetes de la marina en Gatchina, Rusia, e incluso en Río de Janeiro, donde la Universidad lo nombra Doctor Honoris Causa—, el fundador de la Enseñanza universal dentro de poco aparece como una figura del pasado, y la Emancipación intelectual es borrada por la política de educación de las clases pobres, aplicada bajo los auspicios de Guizot y de Victor Cousin por la Monarquía de Julio. Guépin y Bonamy se hacen eco en 1835 de un sentimiento que parece general:

29 L'Enseignemet universal mis à portée de tous les pères de famille, op. cit. 1 .

30 Carta de Jacotot a Lambert del 26 de noviembre de 1835, Museo Nacional de la Educación, Mont-Saint-Aignan. 
El método Jacotot, que hizo furor en Nantes, es común sólo en un establecimiento, dirigido por el Sr. Papote. Pero cualquiera que sean los éxitos extraordinarios obtenidos por este profesor, estamos lejos de atribuir al método un valor que no tiene. Si bien reconocemos que Jacotot ha hecho un gran servicio a la enseñanza, y que sería útil que el espíritu de sus obras se encarnara en algunos de los miembros de la Universidad, no vemos en el célebre profesor de Lovaina más que un hábil demoledor. Cada vez que exclusivamente se trata de reconstruir, Jacotot no vio la enseñanza bajo todas sus caras, también dejó mucho por decir y mucho por hacer ${ }^{31}$.

Este juicio debe ser atenuado, de hecho, a través de los testimonios que manifiestan que, más allá del hecho de una regresión constatable de la Enseñanza universal, el método podría tener, en un futuro cercano, un reconocimiento obligado. Sea la propuesta de Ratier al comité central de la asociación libre por la educación del pueblo, el 14 de septiembre de 1833 , solicitando que doce alumnos que aún no saben nada le sean confiados para hacerlos profesores ${ }^{32}$, o la necrología de AntoineJoseph Sabatier, redactada por Joseph Monrad en 1838, constatando ante los institutores que llegará ciertamente un día en que, examinado con más atención y comprensión, mejor comprendido por el público, [el método de Jacotot] será restituido en la opinión, [y que entonces] veremos que en el fondo no es otra cosa que la puesta en práctica de muchos principios formulados por grandes pensadores, antiguos o modernos, tales como Jenofonte, Sócrates, Bacon, Descartes, Condillac, Rousseau, etc.; y nos sorprenderemos de haber desechado bajo un nombre lo que adoptamos bajo otro ${ }^{33}$.

31 Guépin, Ange et Bonamy, Eugène. Nantes au XIX siècle, Nantes, 1835, reeditado por Ph. Le Pichot et A. Supiot. Université de Nantes, 1981.

32 Ver Le Populaire, periódico de intereses políticos, materiales y morales del pueblo, dirigido por Etienne Cabet, número del 29 de septiembre de 1833, suplemento.

33 L'Echo des écoles primaires, janvier 1838. 543. 
Bajo el Segundo Imperio, la literatura evoca el método a propósito de figuras obsoletas de maestros de escuela de pueblo en tiempos antiguos ${ }^{34}$, pero las publicaciones más modernistas lo colocan en el panteón de la pedagogía francesa ${ }^{35}$. Éste pasa a ser finalmente, desde antes de la reforma de Ferry, precursor del método natural en pedagogía. Un director de escuela normal de Versalles lo cita en sus conferencias a los estudiantes-maestros e institutores después del método de indagación de Sócrates, del método intuitivo de Pestalozzi y del método moral de Girard ${ }^{36}$ : estos son todos preciosos, pero preconizan con su exageración, que les condena a desaparecer junto con sus fundadores. Constituidos por pedagogos de fin del siglo XIX como monumentos, estos métodos son evaluados en función de la política de la instrucción popular obligatoria aplicada por el Estado moderno republicano; y en ese desplazamiento de los desafíos de la instrucción y de la educación, el método ya no tiene por fin la emancipación intelectual de las clases pobres, sino, explícitamente referido a Kant, "la elevación de un niño tan cerca como sea posible de la perfección humana, no solamente en vista de su destino humano, sino por el bien de la humanidad en general" ${ }^{\prime 37}$. No nos sentimos más como Racine y tenemos, a partir de ahora, razón.

34 Ponson du Terrail, Le Nouveau maître d'école, Paris, 1860.

35 Artículo de J. Paroz en L'Ecole Normale, journal de l'enseignement pratique dirigido por Pierre Larousse, $\mathrm{n}^{\circ} 38$ del 23 de julio de 1865.

36 Marlotti, L. Conférences de pédagogie. Manuel des élèves-maîtres et des instituteurs. Paris: Hachette, deuxième édition, 1873.

37 Ver Marion, Henri. "Méthode du Diccionaire de pédagogie de Ferdinand Buisson", op. cit. 1900; Douailler, Stéphane. "La Leçon de morale entre l'éducation et I'instruction". Cahier Aubois d'Histoire de l'Education, 8 (1984) (10 rue Saint-Martin des Aires, 10000 Troyes), y Mauve, Christiane. "Une centenaire mélancolique..." Cahier Philosophiques, CNDP, 6 (1981). 